\title{
The sources of the Kuznets relationship between road fatalities and economic growth
}

\begin{abstract}
This paper reports the results of an empirical analysis of the Kuznets curve relationship between per capita income and road fatalities across 60 countries over the period 1972 ï 2004. This relationship hypothesizes that the number of road fatalities increases with increasing motorization in the early stages of economic growth. Eventually, due to advances in technical, policy and political institutions, it declines as per capita income increases. The quality of political institutions as well as improvements in medical care and technology are hypothesized to impact road fatalities. Results indicate evidence of a Kuznets curve relationship between per capita income and road fatalities for both highly developed and less developed countries and support our hypothesis that changes in institutional quality and medical improvements underlie the Kuznets relationship. The evidence presented in this study suggests that lowering corruption levels as well as improvements in medical care and technology would help to reduce road fatalities.
\end{abstract}

Keyword: Medical care and technology; Negative binomial model; Kuznets curve; Corruption; Political rights 